\title{
Assessment of the levels of nitric oxide (NO) and cytokines (IL-5, IL-6, IL-13, TNF, IFN-gamma) in giardiosis
}

\author{
Joanna Matowicka-Karna ${ }^{1}$, Maciej Kralisz ${ }^{2}$, Halina Kemona ${ }^{1}$ \\ ${ }^{1}$ Department of Clinical Laboratory Diagnostics, Medical University of Bialystok, Poland \\ ${ }^{2}$ III Department of Internal Diseases and Gastroenterology, the J. Sniadecki Provincial Hospital \\ in Bialystok, Poland
}

\begin{abstract}
The current study aims to determine the involvement of cellular responses in combating Giardia intestinalis invasion. The study group consisted of 44 women and 18 men, aged 18-72 years, infected with $G$. intestinalis. The diagnosis was established based on laboratory investigations (examination of stool, choloscopy, GSA-65). Blood for analysis was collected before antiparasitic treatment and two weeks after treatment termination. The control group consisted of 22 women and 18 men aged 20-45 years. The serum concentrations of IL-5, IL-6, IL-13, TNF, IFN- $\gamma$ were assayed using a set of Quantikine human. The concentrations of NO in the serum were determined using a set of Total Nitric Oxide Assay. Patients infected with G. intestinalis showed a statistically significant increase in the levels of NO, IFN- $\gamma$ and IL-13. Even the antiparasitic treatment applied did not reduce the levels of these parameters and only caused a rise in IL-6. Our study showed a lack of acute inflammatory state in the course of G. intestinalis infection. (Folia Histochemica et Cytobiologica 2011; Vol. 49, No. 2, pp. 280-284)
\end{abstract}

Key words: giardiosis, IL-5, IL-6, IL-13, TNF-alpha, IFN-gamma, NO

\section{Introduction}

Parasitic invasions may trigger local or systemic inflammatory processes caused both by parasitic antigens and exotoxins [1, 2]. Antibody-dependent cell cytotoxicity (ADCC), with eosinophils as the effector cells, is the major mechanism of antiparasitic defense. The antibody-dependent antiparasitic immunity involves a few mechanisms that cause blockage of the receptors on the parasite surface, induce direct damage of the parasite through activation of the complement system, or increase IgE production.

\footnotetext{
Correspondence address: J. Matowicka-Karna, Department of Clinical Laboratory Diagnostics, Medical University of Bialystok,

J. Waszyngtona Str. 15a, 15-269 Bialystok, Poland; tel./fax: (+48 85) 74685 84;

e-mail: matowic@umwb.edu.pl
}

Jimenez et al. [3], who studied the effect of excretory and secretory antigens of $G$. intestinalis, observed an increase in total IgE level as well as systemic and local stimulation of IgA secretion. Earlier, Zhou et al. [4] had found IgA to play an important part in G. intestinalis invasion [5]. IL-6, which is involved in the immune response, inflammatory reaction and hemopoiesis, and stimulates the acute phase of protein synthesis, one of the factors that regulate defense mechanisms. In the course of giardiosis in mice, an increase was observed in IL- 6 and in the production of IFN- $\gamma$ by $\mathrm{CD}^{+}{ }^{+}$lymphocytes [6]. Some authors have suggested that in response to Giardia antigen, T cells release no lymphocytes-specific cytokines, whereas mast cells release cell mediators, prostaglandins and kinin proteinase [7]. Sometimes giardiosis is asymptomatic (carrier state), whereas high invasiveness of G. intestinalis has been shown to occur in the case of $\operatorname{IgG}$ and $\operatorname{IgA}$ deficiency $[2,8]$. 
Apart from IL-6, IL-5 also contributes to a humoral response. This cytokine is produced by Th 2 lymphocytes, and takes part in growth induction and differentiation of B- and T-cells. IL-5 stimulates the proliferation and differentiation of eosinophil precursors, stimulates their degranulation and production of reactive oxygen compounds. It exerts a chemotactic effect on eosinophils and induces eosinophilia $[9,10]$.

Another cytokine secreted by Th2 lymphocytes is IL-13, which by augmenting proliferation of activated B-cells exerts an effect on monocytes and B lymphocytes. Its major physiological role is to regulate antiparasitic response. It inhibits antibody-dependent cytotoxicity and secretion of proinflammatory cytokines (TNF, IL-1, IL-6 and IL-8) [11].

TNF (secreted by macrophages) and IFN- $\gamma$ (secreted by T- and NKT-cells) contribute to the increased expression of inducible nitric oxide synthase (iNOS) in, for example, vascular endothelial cells. IFN- $\gamma$, TNF and IL-1 induce NO generation in macrophages. Nitric oxide is a product of $\mathrm{L}$-arginine metabolism and is one of the oxygen-independent toxins. NO regulates the immune response and is responsible for macrophage cytotoxicity [12].

G. intestinalis is removed from the alimentary tract by antibodies that bind with the parasite surface antigens. The released inflammatory mediators lead to parasite damage, accelerate intestinal peristalsis, and facilitate parasite eradication by the production of mucus in the intestine, which is indispensable for parasite removal [13]. The current study aimed to determine the involvement of humoral and cellular responses in combating $G$. intestinalis invasion. The humoral response was assessed by determining the levels of IL-5, IL-6 and IL-13, whereas the cellular response was evaluated by measuring IFN- $\gamma$ and TNF.

The study objective was to find out:

1. whether $G$. intestinalis infection affects the parameters studied, and

2. whether the antiparasitic treatment applied changed the immune response.

\section{Material and methods}

The study involved 62 patients (aged 18-72 years) infected with Giardia intestinalis, 44 women and 18 men (G1). The patients were hospitalized in III Department of Internal Diseases and Gastroenterology at the J. Sniadecki Provincial Hospital in Bialystok. Giardiosis was diagnosed based on the clinical picture (acute abdominal pains, diarrhea, reduction of strength, sub-febrile temperature) parasitological examination of feces, enzyme immunoassay of feces for detection of specific protein GSA-65 (Wampol Giardia II, TECHLAB, USA) and microscopic examination of duodenal contents. The patients were treated with metronidazole (c. ten days), tinidazole (two days), cholagogues (cholestil, cholamide) and bioregulators of physiological intestinal flora (lakcid). Blood for analysis was collected prior to treatment (subgroup G1) and two weeks after antiparasitic treatment (subgroup G2: 25 patients, 16 women and nine men). The effectiveness of anti-parasitic treatment was assessed by determining titers of GSA- 65 protein in the feces and by testing the bile for trophozoites.

The control group (C) consisted of 40 healthy subjects (aged 20-45 years), 22 women and 18 men. All the parameters examined in the study group and the control group with regard to age and gender were subjected to statistical analysis.

According to the Guidelines for Good Clinical Practice, all the patients gave consent for participation in the study. The study was approved by the Bioethics Committee, Medical University of Bialystok.

The serum levels of IL-5, IL- 6 and IL-13, TNF and IFN- $\gamma$ were determined by the ELISA method using a Quantikine human kit (R\&D Systems, USA). The level of NO in the sera was determined by the calorimetric method using a Total Nitric Oxide Assay kit (R\&D Systems, USA).

The results were subjected to statistical analysis using the Statistica 8.0 program. The $t$-Student test was used to calculate the features consistent with normal distribution.

\section{Results}

Our study revealed that in patients infected with G. intestinalis (G1), the mean levels of IL-5 and IL-6 were statistically significantly lower than those observed in healthy subjects $(\mathrm{p}<0.05)$ (Table 1$)$. After antiparasitic treatment (G2), the mean level of IL-5 was increased, but was not as high as in the control group (C) (Table 1). However, the level of IL-6 in patients after treatment $(\mathrm{G} 2)$ was found to increase statistically significantly compared to subgroup G1 and group C (Table 1). For both the study and the control groups, the leukocyte and eosinophil count was found to be within the normal range (data not shown).

In patients infected with $G$. intestinalis (G1), the mean level of IL-13 was six times higher than the value obtained in healthy subjects (group C) $(\mathrm{p}<0.001)$. After the antiparasitic treatment (G2), the value still remained the same as in subgroup G1 (Table 1).

The mean level of TNF in patients infected with G. intestinalis (G1) was similar to that observed in subgroup G2 (after antiparasitic treatment). Although the values obtained in these two subgroups were twice as high as in the control group, the differences were not statistically significant (Table 1).

In patients infected with $G$. intestinalis (G1), the mean level of IFN- $\gamma$ was more than three times higher than that noted in healthy subjects (group C). 
Table 1. Concentrations of chosen parameters assessing the immune response in patients infected with $G$. intestinalis prior to treatment (G1), two weeks after termination of antiparasitic treatment (G2) and in a control group (C). Statistical analysis was performed in examined groups. Values of $\mathrm{p}<0.05$ were considered to be significant

\begin{tabular}{|c|c|c|c|c|c|}
\hline Parameters & $\begin{array}{l}\text { Study subgroup G1 } \\
\mathrm{n}=62 \mathrm{X} \pm \mathrm{SD}\end{array}$ & $\begin{array}{c}\text { Study subgroup G2 } \\
\mathrm{n}=25 \mathrm{X} \pm \mathrm{SD}\end{array}$ & $\begin{array}{l}\text { Control group C } \\
n=40 X \pm S D\end{array}$ & & $\mathbf{p}$ \\
\hline IL-5 & $2.27 \pm 1.14$ & $2.59 \pm 1.38$ & $3.59 \pm 1.59$ & $\begin{array}{l}\text { G1:G2 } \\
\text { G1:C } \\
\text { G2:C }\end{array}$ & $\begin{array}{l}0.2<\mathrm{p}<0.3 \\
\mathrm{p}<0.05^{*} \\
\mathrm{p}<0.05^{*}\end{array}$ \\
\hline IL-6 & $1.59 \pm 3.12$ & $4.06 \pm 8.78$ & $2.45 \pm 1.44$ & $\begin{array}{l}\text { G1:G2 } \\
\text { G1:C } \\
\text { G2:C }\end{array}$ & $\begin{array}{l}\mathrm{p}<0.05^{*} \\
\mathrm{p}<0.05^{*} \\
\mathrm{p}<0.05^{*}\end{array}$ \\
\hline IL-13 & $42.37 \pm 27.79$ & $41.41 \pm 31.07$ & $7.00 \pm 3.91$ & $\begin{array}{l}\text { G1:G2 } \\
\text { G1:C } \\
\text { G2:C }\end{array}$ & $\begin{array}{l}0.5<\mathrm{p}<0.6 \\
\mathrm{p}<0.001^{*} \\
\mathrm{p}<0.001^{*}\end{array}$ \\
\hline TNF & $2.03 \pm 1.74$ & $1.82 \pm 1.30$ & $0.91 \pm 0.48$ & $\begin{array}{l}\text { G1:G2 } \\
\text { G1:C } \\
\text { G2:C }\end{array}$ & $\begin{array}{l}0.6<p<0.7 \\
0.05<p<0.1 \\
0.1<p<0.2\end{array}$ \\
\hline IFN- $\gamma$ & $25.91 \pm 13.79$ & $24.51 \pm 12.36$ & $7.00 \pm 3.91$ & $\begin{array}{l}\text { G1:G2 } \\
\text { G1:C } \\
\text { G2:C }\end{array}$ & $\begin{array}{l}0.8<\mathrm{p}<0.9 \\
\mathrm{p}<0.001^{*} \\
\mathrm{p}<0.001^{*}\end{array}$ \\
\hline NO & $35.16 \pm 11.27$ & $38.27 \pm 14.24$ & $22.53 \pm 3.15$ & $\begin{array}{l}\text { G1:G2 } \\
\text { G1:C } \\
\text { G2:C }\end{array}$ & $\begin{array}{l}0.5<\mathrm{p}<0.6 \\
\mathrm{p}<0.001^{*} \\
\mathrm{p}<0.001^{*}\end{array}$ \\
\hline
\end{tabular}

Following the antiparasitic treatment (G2) the level of IFN- $\gamma$ was decreased as compared to the value observed in subgroup G1, although the difference between G1 and $\mathrm{G} 2$ was not statistically significant (Table 1 ).

The mean level of nitric oxide in patients infected with $G$. intestinalis (G1) was statistically significantly higher than the value obtained in the control group $(\mathrm{p}<0.001)$. The antiparasitic treatment (G2) caused its increase, although the difference was not statistically significant as compared to G1 (Table 1).

\section{Discussion}

Both humoral and cellular mechanisms are involved in combating parasitic invasions. The humoral response involves antibodies, whereas in the cellular response T-cells and secreted cytokines react directly with parasitic antigens.

G. intestinalis may impair the immune response of the host organism, something which has been documented in animal models [5]. In humans, infection with this parasite causes a reduction in the levels of class IgG and IgA antibodies. The immune reaction in $G$. intestinalis invasion consists in the production of antibodies and release of inflammatory mediators that lead to parasite damage. IgA plays a major role as it contributes to the elimination of this parasite from the alimentary tract. TNF and IL-1 (products of nonspecific inflammatory reaction) cause stimula- tion of goblet cells and production of mucus which facilitates removal of dead parasites [2,13].

Apart from antibodies, the mechanisms that lead to inactivation and death of parasites also involve nitric oxide. NO is generated in the reaction initiated by nitric oxide synthase with the involvement of L-arginine, oxygen $\left(\mathrm{O}_{2}\right)$, NADPH and tetrahydrobiopterin. NO is a very important mediator of many biological processes, e.g. it is involved in killing microorganisms, parasites and cancer cells. NO and arginine are responsible for the defense of the host against $G$. intestinalis infection. NO inhibits encystation of trophozoites and excystation of $G$. intestinalis cysts. The parasite defends itself by inhibiting NO production in intestinal epithelial cells using arginine which is the basic substrate in NO production [14]. Ringqvist et al. [15] reported a study with recombinant arginine deiminase, in which proteins secreted by Giardia could impair innate immune mechanisms, e.g. NO production, whereas a direct contact of Giardia with intestinal epithelial cells leads to the release of enzymes by the parasite, which facilitate intestinal colonization.

We found that parasitic infection stimulated NO production, which was not reduced even by the antiparasitic treatment applied. Eckmann et al. [16] described the cytotoxic and cytostatic actions of NO on $G$. intestinalis trophozoites in in vitro conditions. The increased NO production can successively reduce the 
number of parasites, and the balance maintained between NO and pro- and anti-inflammatory cytokines definitely determines morbidity and the efficacy of the immune response [17]. In addition to NO, the action of bactericidal proteins, such as alpha-defensins or lactopherin produced in the alimentary tract, is another important mechanism involved in the immunological fight against the parasite $[18,19]$.

In the current study, in the course of giardiosis, the level of IL-13 increased statistically significantly (six-fold) as compared to that observed in healthy subjects. IL-13 is known to increase antiparasitic immunity and IgE production, at the same time decreasing secretion of proinflammatory cytokines such as IL-6, TNF, IL-1, IL-8 and inhibiting ADCC. This may be why the level of IL- 6 assessed in the course of giardiosis was statistically lower compared to the values obtained in the control group. Many authors have indicated the changes in the levels of immunological indices in parasitic invasions, e.g. the observed increase in IL-5 characteristic of parasitosis [9]. Research previously performed by the authors on patients infected with $G$. lamblia demonstrated a statistically significant increase in the concentration of IL-5 [20]. In the course of acute form of Schistosoma mansoni infection, both the level of IL-5 and that of IFN- $\gamma$ showed a marked rise [21]. Brattig et al. [11] revealed an increase in IL-5 and IL-13 in response to administration of the extract of soluble antigen of Onchocerca volvulus. The early stage of Onchocerca volvulus infection described by Cooper et al. [22] caused increased production of IL-5 and IFN- $\gamma$.

Ajdary et al. [23] in patients chronically infected with Leishmania (but untreated) observed a statistically significant increase in the levels of IL-5, IFN- $\gamma$ and IL-13 produced by peripheral blood mononuclear cells (PBMC) in in vitro conditions. These findings suggest the occurrence of mixed type Th1/Th2 response. However, patients with acute disease showed Th1 response, which was indicated by increased concentration of IFN- $\gamma$ and low levels of IL-5 and IL-13.

Ishikawa et al. [24] described experimental infection of mice with roundworms: Trichinella spiralis and Nippostrongylus brasiliensis, in response to which cytokines (among others IL-5 and IFN- $\gamma$ ) released from Th1 and Th 2 cells are involved. IFN $-\gamma$ produced by activated Th1 cells shows a suppressive action on the synthesis and release of IgE. By inducing the production of reactive oxygen and secretion of hydrogen peroxide, IFN- $\gamma$ stimulates intracellular killing of parasites by macrophages [25]. Touil-Boukoffa et al. [26] have revealed that the defense mechanisms in the course of echinococcosis involve, apart from IFN- $\gamma$, also IL-6. A study conducted by Ajami and Rafiei [27] in patients infected with Hymenolepis nana showed an increase in the levels of IL-5, IL-12, IL-13 and IFN- $\gamma$. We found elevated levels of IL-13 and IFN- $\gamma$ in patients infected with $G$. intestinalis. Th1 lymphocytes produce IFN- $\gamma$, which inhibits proliferation and the action of Th2 cells, and assist in the cellular type response. Th2 lymphocytes generate IL-4, IL-5, IL-6, IL-10 and IL-13, and promote the humoral response.

Elevated levels of IL- 6 are observed in the course of many parasitoses due to accompanying inflammation. IL-6 is a sensitive and early, though nonspecific, marker of inflammatory reaction [28]. It exerts a pyrogenic effect, together with IL-1, TNF and IFN, by elevating body temperature and stimulating the production of prostaglandins [26]. We found no increase in the level of C-reactive protein in giardiosis, despite the fact that IL- 6 stimulates the production of acute phase proteins (data not shown). However, the antiparasitic treatment in patients infected with $G$. intestinalis caused a statistically significant increase in the level of IL-6, but had no effect on IL-5.

Bayraktar et al. [29] have shown that G. intestinalis infection causes an increase in the levels of TNF and sIL-2R. However, a rise was observed in the levels of IL-1 $\beta$, IL-6, IL-8, NO and CRP when allergic symptoms accompanied parasitosis [30]. In our study, an increase was found in NO only, but there were no symptoms of allergy and the levels of IL-6 and CRP were not elevated. According to Zhou et al. [31], IL-6 and TNF have a great effect on $G$. intestinalis invasion in mice. These are proinflammatory cytokines which in the course of Giardia infection increase intestinal epithelial permeability.

In our study, the level of TNF was slightly elevated (both before and after treatment) and showed minimal statistically insignificant differences as compared to the values obtained in healthy subjects. The production and release of TNF are stimulated by lipopolysaccharide and IFN- $\gamma$, whereas IL-13 is one of the inhibitors of its secretion. TNF and IFN- $\gamma$ enhance the expression of inducible nitric oxide synthase, and increased NO secretion leads to parasite eradication. If IL-13 inhibits TNF secretion, then NO generation can only be increased by IFN- $\gamma$.

Our own investigations, as well as literature data, indicate the occurrence of the immune and inflammatory responses in the course of parasitic invasions, when IFN- $\gamma$, TNF, IL- $1 \beta$, IL-5 and IL-13 are released $[8,23]$. Our findings may suggest that $G$. intestinalis infection is not accompanied by acute inflammatory state. The current study seems to confirm the occurrence of both Th1 and Th 2 response in giardiosis. This 
parasitic invasion is accompanied by chronic inflammation and the antiparasitic treatment applied seems to cause an increase in the level of IL-6, but does not affect the other parameters.

\section{Conclusions}

An increased level of nitric oxide in the course of giardiosis seems to confirm its involvement in the regulation of immune response. Increased levels of IL-13 and IFN- $\gamma$ in the course of giardiosis indicate substantial immunization of the host organism. Antiparasitic treatment in giardiosis does not lead to normalization of the parameters studied.

\section{References}

1. Adam RD. Biology of Giardia lamblia. Clin Microbiol Rev. 2001;3:447-475.

2. Faubert G. Immune response to Giardia duodenalis. Clin Microbiol Rev. 2000;13:35-54.

3. Jimenez JC, Fontaine J, Grzych J-M, Dei-Cas E, Capron M. Systemic and mucosal responses to oral administration of excretory and secretory antigens from Giardia intestinalis. Clin Diagn Lab Immunology. 2004;11:152-160.

4. Zhou P, Li E, Robertson J, Nash T, Singer SM. Role of interleukin-6 in the control of acute and chronic Giardia lamblia infections in mice. Infect Immun. 2003;71:1566-1568.

5. Langford TD, Housley MP, Boes M et al. Central importance of immunoglobulin A in host defense against Giardia spp. Infect Immun. 2002;70:11-18.

6. Ebert EC. Giardia induces proliferation and interferon production by intestinal lymphocytes. Gut. 1999;44:342-346.

7. Singer SM, Nash TE. T-cell-dependent control of acute Giardia lamblia infections in mice. Infect Immun. 2000;68:170-175.

8. Majewska AC. Doświadczalna giardioza: badania porównawcze biologicznych właściwości izolatów Giardia. Wyd Kat Biol Parazytol Lek AM, 1996; Poznań.

9. Faccioli LH, Vargaftig BB, Medeiros A, Malheiros A. Cytokines in the modulation of eosinophilia. Mem Inst Oswaldo Cruz Rio de Janeiro. 1997; 92, suppl.II:109-114.

10. Weltman JK. Cytokines: regulators of eosinophilic inflammation. Allergy Asthma Proc. 2000;21:203-207.

11. Brattig NW, Lepping B, Timmann C et al. Onchocerca volvulus-exposed persons fail to produce interferon-gamma in response to $\mathrm{O}$. volvulus antigen but mount proliferative responses with interleukin-5 and IL-13 production that decrease with increasing microfilarial density. J Infect Dis. 2002;185: 1148-1154.

12. Li E, Zhou P, Singer SM. Neuronal nitric oxide synthase is necessary for elimination of Giardia lamblia infections in mice. J Immunol. 2006;176:516-521.

13. Hawrelak J. Giardiasis: pathophysiology and management. Altern Med Rev. 2003;8:129-142.

14. Eckmann L, Laurent F, Langford D et al. Nitric oxide production by human intestinal epithelial cells and competition for arginine as potential determinants of host defense against the lumen-dwelling pathogen Giardia lamblia. J Immunol. 2000;164:1478-1487.

15. Ringqvist E, Palm JED, Skarin $\mathrm{H}$ et al. Release of metabolic enzymes by Giardia in response to interaction with intestinal epithelial cells. Molecul Biochem Parasitol. 2008;159:85-91.

16. Eckmann L. Mucosal defences against Giardia. Parasite Immunol. 2003;25:259-270.

17. Brunet LR. Nitric oxide in parasitic infections. Int Immunopharmacol. 2001;1:1457-1467.

18. McDonald V. Parasites in the gastrointestinal tract. Parasite Immunol. 2003;25:231-234.

19. Roxstrom-Lindquist K, Palm D, Reiner D, Ringqvist E, Svard SG. Giardia immunity - an update. Trends Parasitol. 2006;22:26-31.

20. Matowicka-Karna J, Dymicka-Piekarska V, Kemona H. IFN-gamma, IL-5, IL-6 and IgE in patients infected with Giardia intestinalis. Folia Histochem Cytobiol. 2009;47:93-97.

21. de Jesus AR, Silva A, Santana LB et al. Clinical and immunologic evaluation of 31 patients with acute schistosomiasis mansoni. J Infect Dis. 2002;185:98-105.

22. Cooper PJ, Mancero T, Espinel M et al. Early human infection with Onchocerca volvulus is associated with an enhanced parasite-specific cellular immune response. J Infect Dis. 2001;183:1662-1668.

23. Ajdary S, Riazi-Rad F, Alimohammadian M-H, Pakzad S-R. Immune response to Leishmania antigen in anthroponotic cutaneous leishmaniasis. J Infect. 2009; doi:10.1016/j.jinf. 2009.05.010.

24. Ishikawa N, Goyal PK, Mahida YR, Li K-F, Wakelin D. Early cytokine responses during intestinalis parasitic infections. Immunology. 1998;93:257-263.

25. Lucey DR, Clerici M, Shearer GM. Type 1 and type 2 cytokine dysregulation in human infectious, neoplastic, and inflammatory diseases. Clin Microbiol Rev. 1996; 9:532-562.

26. Touil-Boukoffa C, Sanceau J, Tayebi B, Wietzerbin J. Relationship among circulating interferon, tumor necrosis factoralpha, and interlekin- 6 and serologic reaction against parasitic antigen in human hydatidosis. IInterferon Cytokine Res. 1997;17:211-217.

27. Ajami A, Rafiei A. Cytokine production in Hymenolepis nana infection. Iran J Immunol. 2007;4:236-240.

28. Bienz M, Dai WJ, Welle M, Gottstein B, Muller N. Interleukin-6-deficient mice are highly susceptible to Giardia lamblia infection but exhibit normal intestinal immunoglobulin A responses against the parasite. Infect Immun. 2003; 71:1569-1573.

29. Bayraktar MR, Mehmet N, Durmaz R. Serum cytokine changes in Turkish children infected with Giardia lamblia with and without allergy: effect of metronidazole treatment. Acta Tropica. 2005;95:116-122.

30. Di Prisco MC, Hagel I, Lynch NR et al. Association between giardiasis and allergy. Ann Allergy Asthma Immunol. 1998;81:261-265.

31. Zhou P, Li E, Shea-Donohue T, Singer SM. Tumor necrosis factor á contributes to protection against Giardia lamblia infection in mice. Parasite Immunol. 2007;29: 367-374.

Submitted: 8 June, 2010

Accepted after reviews: 30 January, 2011 